\title{
RECYCLED PULSARS AND LOW MASS X-RAY BINARIES
}

\author{
G. S. Bisnovatyi-Kogan \\ Space Research Institute
}

\begin{abstract}
A magnetized neutron star may appear as a radio pulsar or an X-ray source. The latter is connected with a binary system where accretion from a normal star onto the neutron star produces $\mathrm{X}$-ray emission. At the end of the evolution of a normal non-massive star, accretion stops and the neutron star becomes a recycled radio pulsar. Further evolution may lead to an additional transition from a radio pulsar to a low mass X-ray binary (LMXB). The formation of a single recycled pulsar is considered and a new mechanism of "enhanced evaporation" in globular clusters is analyzed.
\end{abstract}

\section{[ntroduction}

ladio pulsars in binary systems and millisecond ralio pulsars are united in one class of recycled pulars whose rotation has been accelerated during a hase of disc accretion in a binary system with an K-ray source. There is a difference between low nass and high mass X-ray binaries. Most X-ray ulsars belong to high mass binaries. In wide bilaries of this type long period ( $P \geq 100 \mathrm{~s}$ ) X-ray ulsars are formed, which will not be seen after the nd of accretion, because of slow rotation. In the lose high mass binaries the neutron star may rotate ather rapidly, but the mass transfer to the neutron tar in such a system in the last stages of evolution nay be so high that the mass of the neutron star vill exceed the limiting mass and a black hole will re formed. Another possible outcome is formation if two neutron stars in the binary system.

Formation of recycled pulsars most likely hapsens in LMXB. There is a strong concentration of $\mathrm{MXB}$ sources in globular clusters; the same type of :oncentration in the distribution of recycled pulsars :onfirms the genetic connection between LMXB and ecycled pulsars.

\section{Observational data}

jystematic searches for recycled pulsars in globular :lusters has led to a continuous increase of their uumber. There are 31 recycled pulsars known as of lune 1990, they can be divided into three groups see table 1).

The first group of 14 pulsars consists of binaries vith white dwarf companions. The second of 14 nembers contains single radio pulsars; in the third ;roup there are 3 binaries consisting of two neutron itars.

Acceleration of neutron-star rotation occurs in ihe LMXB disc accretion stage. The evolutionary icheme for the recycled pulsars is given in figure 1 aken from Bisnovatyi-Kogan (1989).

\section{Evolution of isolated binary pulsars}

There are three main evolutionary effects on an isolated binary system

a) orbital angular momentum losses due to gravitational radiation (Landau and Lifshitz 1962),

b) variation of the binary period during the mass transfer, when the companion star fills its Roche lobe (Paczyński 1981) at the stage of LMXB,

c) magnetodipole losses and breaking of rotation of the neutron star as a radio pulsar (Landau and Lifshitz 1962).

These evolutionary factors imply the following evolutionary paths of the binaries. Evolution of a newly formed recycled pulsar will be very slow when the binary period exceeds 8 hours. For such a period gravitational radiation changes the parameters of the binary on a time larger than the cosmological time $\tau=2 \times 10^{10}$ years. For smaller binary periods, gravitational radiation leads to a decrease of the distance between stars in binaries in a shorter time. If the companion of the neutron star is a white dwarf, then it once more fills its Roche lobe and mass transfer begins for the second time, leading to formation of a second generation LMXB (Bisnovatyi-Kogan 1989). The binary period of a second generation LMXB may be several minutes, and the companion of the neutron star in such system will be a white dwarf consisting of elements not lighter than helium. An example of such a system is the LMXB $4 U 1820-30$ with an orbital period of 11 minutes and a white dwarf companion. The recycled pulsar PSR 0021-72A in 47 Tuc with an orbital period 0.022 days may be considered as a predecessor of a second generation LMXB.

When a system of two neutron stars has a small period like PSR $1913+16$ or $2127+11 C$, gravitational radiation leads to their approach toward each other. At the end of this evolution one expects a rapid nonstationary process of mass transfer, possibly an explosion and/or merging of the two neu- 
Table 1 Recycled radio pulsars

\begin{tabular}{|c|c|c|c|c|c|c|c|c|c|}
\hline$\overline{\mathrm{Nr}}$ & $\begin{array}{l}\text { Pyilsar } \\
\text { PSR }\end{array}$ & $\begin{array}{l}P \\
(\mathrm{~ms})\end{array}$ & $\begin{array}{l}\tau= \\
P / 2 \dot{P}(y)\end{array}$ & $\begin{array}{l}\log B \\
(G)\end{array}$ & $\begin{array}{l}P_{\text {orb }} \\
\text { (d) }\end{array}$ & $e$ & $\begin{array}{l}f(M) \\
\left(\mathrm{M}_{\odot}\right)\end{array}$ & $M_{2} / M_{\odot}$ & $\begin{array}{l}\text { Globular } \\
\text { cluster }\end{array}$ \\
\hline 1 & $0221-72 B$ & 6.1 & & & 7.95 & & & & 47 Tuc \\
\hline 2 & $0655+64$ & 196 & $5 \times 10^{9}$ & 10 & 1.03 & $7.5 \times 10^{-6}$ & 0.0712 & $0.7-1.3$ & \\
\hline 3 & $0820+02$ & 865 & $10^{8}$ & 11.5 & 1232 & 0.0119 & 0.003 & $0.2-0.4$ & ? \\
\hline 4 & $1516+02 B$ & 7.9 & & & few & & & & $\operatorname{M5}\left(\Delta v_{\text {orb }}<20 \mathrm{~km} / \mathrm{s}\right)$ \\
\hline 5 & $1620-26$ & 11.1 & $2 \times 10^{8}$ & 9.5 & 191 & 0.025 & 0.008 & $\sim 0.35$ & M4 \\
\hline 6 & $1802-07$ & 23.1 & & & 2.62 & & & & NGC 6539 \\
\hline 7 & $1820-11$ & 279.8 & $3.2 \times 10^{6}$ & 11.8 & 357.8 & 0.795 & 0.068 & & \\
\hline 8 & $1831-00$ & 521 & $10^{\circ}$ & 10.9 & 1.81 & 0.0001 & $1.2 \times 10^{-4}$ & $.06-.13$ & \\
\hline 9 & $1855+09$ & 5.4 & $4 \times 10^{9}$ & 8.5 & 12.33 & $2 \times 10^{-8}$ & 0.0056 & $0.2-0.4$ & \\
\hline 10 & $1908+00$ & 3.6 & & & few & & & & NGC 6760 \\
\hline 11 & $1953+29$ & 6.1 & $3 \times 10^{2}$ & 8.6 & 117 & $3.3 \times 10^{-4}$ & 0.0024 & $0.2-0.4$ & \\
\hline 12 & $1744-24 A$ & 11.6 & & & 0.0756 & $<10^{-3}$ & $3.2 \times 10^{-4}$ & $>0.1$ & Ter $5^{(e c l)}$ \\
\hline 13 & $1957+20$ & 1.6 & $1.7 \times 10^{\circ}$ & 8.2 & 0.38 & $<10^{-3}$ & $5.2 \times 10^{-6}$ & $>0.02$ & (ecl) \\
\hline 14 & $0021-72 A$ & 4.5 & & & 0.022 & 0.33 & $1.6 \times 10^{-8}$ & 0.02 & 47 Tuc II \\
\hline 1 & $0021-72 \mathrm{C}$ & 5.757 & & & & & & & 47 Tuc $^{\dagger}$ \\
\hline 2 & $0021-72 D$ & 5.35 & & & & & & & 47 Tuc $^{t}$ \\
\hline 3 & $1310+18$ & 33 & & & & & & & M53 \\
\hline 4 & $1516+02 \mathrm{~A}$ & 5.5 & & & & & & & M5 \\
\hline 5 & $1639+36$ & 10.4 & & & & & & & M13 \\
\hline 6 & $1745-20$ & 288.6 & & & & & & & NGC $6440^{\dagger}$ \\
\hline 7 & $1820-30 \mathrm{~A}$ & 5.44 & & & & & & & NGC $6624^{\dagger}$ \\
\hline 8 & $1820-30 B$ & 378.6 & & & & & & & NGC $6624^{\dagger}$ \\
\hline 9 & $1821-24$ & 3.1 & $3 \times 10^{7}$ & 9.3 & & & & & M28 \\
\hline 10 & $1937+21$ & 1.6 & $3 \times 10^{8}$ & 8.6 & & & & & \\
\hline 11 & $2127+11 A$ & 111 & $\sim 10^{8}$ & $\sim 10.7$ & & & & & M15 \\
\hline 12 & $2127+11 B$ & 56 & $10^{8}$ & 10.3 & & & & & $\mathrm{M}^{\circ} 5^{\diamond}$ \\
\hline 13 & $2127+11 \mathrm{D}$ & 4.65 & & & & & & & $\mathrm{M}^{\ddagger} 5^{\ddagger}$ \\
\hline 14 & $2127+11 I$ & 4.8 & & & & & & & $\mathrm{M}^{\ddagger} 5^{\ddagger}$ \\
\hline 1 & $1913+16$ & 59 & $10^{8}$ & 10.3 & 0.32 & 0.6171 & 0.1322 & 1.4 & \\
\hline 2 & $2127+11 C$ & 30.5 & & & 0.335 & 0.68 & 0.15 & & M15 \\
\hline 3 & $2303+46$ & 1066 & $4 \times 10^{7}$ & 11.8 & 12.34 & 0.6584 & 0.2463 & $1.2-1.5$ & $\left(m_{\mathrm{R}}>26\right)^{?}$ \\
\hline
\end{tabular}

The first group of 14 pulsars consists of binaries with a white dwarf companion. The next 14 are single radio pulsars. The last 3 are members of binaries consisting of two neutron stars. ? - probably not recycled, ecl - eclipsed radio pulsar, II - will transform into second generation LMXB, $\downarrow-$ R. Manchester (1990), †-IAU Circ. 4892, 4808, 4904, $\diamond$ - Anderson et al. (1090)

tron stars (Lattimer and Schramm 1979, Clark and Eardley 1976, Blinnikov et al. 1984).

\section{Formation of a single recycled pulsar}

The discovery of two eclipsing radio pulsars, where matter of the companion is evaporated by the pulsar beam, shows that this process may be responsible for formation of some of the single recycled pulsars (Kluźniak et al. 1988). It may be seen from table 1 that only one of the 14 single recycled pulsars is situated outside of the globular clusters, whereas among the binary objects about half of them are situated outside of globular clusters. This fact may be considered as a strong indication of the importance of stellar collisions for the stripping of the companion. Numerical experiments show that direct stripping of the companion may happen only if the binary period exceeds 10-100 days for the most dense globular clusters (Rappaport, Putney, and Verbunt 1989). The rapid rotation of the single recycled pulsar could be gained only if the binary periods were much smaller.

Mass transfer in LMXBs leads to decrease of the mass of the companion, and one may hope for total absorption of the companion by the neutron star. Calculations accounting for gravitational radiation and mass transfer in the binary when the companion fills its Roche lobe show that during cosmological time $\tau=2 \times 10^{10}$ years the mass of the companion will not become less then $10^{-2} \mathrm{M}_{\odot}$ for normal composition and $0.003-0.0015 \mathrm{M}_{\odot}$ for a companion consisting of heavy elements, with an orbital period between 1.5 and 2.5 hours. The calculations have been done by Bisnovatyi-Kogan (1990) using a realistic equation of state of Zapolsky and Salpeter (1969). One may expect formation of a neutron star with a giant planet companion as a result of such evolution.

When a binary system is a member of a globular cluster, the collisions with surrounding stars lead to an additional mechanism of evaporation of 




Figure 1 Scheme of evolutionary formation of recycled pulsars.

the companion, so called "enhanced evaporation" (Bisnovatyi-Kogan 1990). It is well known from theory (Gurevich and Levin 1950) and numerical experiments (Aarseth and Lecar 1975) that collisions of the binary with surrounding stars lead to additional tightening of the binary if initially it was sufficiently close. For neutron stars with dwarf companions the kinetic energy of the dwarf in the binary $M_{\mathrm{d}} v_{\mathrm{d}}^{2} / 2$ must be larger than the average kinetic energy of the stars $M_{\mathrm{cl}}\left\langle v_{\mathrm{cl}}^{2}\right\rangle / 2$ for further tightening

$$
v_{\mathrm{d}}=\frac{2 \pi R_{12}}{P} \frac{M-M_{\mathrm{d}}}{M}>\left\langle v_{\mathrm{cl}}\right\rangle \sqrt{\frac{M_{\mathrm{cl}}}{M_{\mathrm{d}}}}
$$

When $v_{\mathrm{d}} \ll\left\langle v_{\mathrm{cl}}\right\rangle>\sqrt{M_{\mathrm{cl}} / M_{\mathrm{d}}}$ the dwarf gains energy during collisions and the binary is disrupted during a time comparable with the relaxation time of the dwarf in the cluster (Spitzer 1987)

$$
\tau_{\mathrm{s}}=v_{\mathrm{d}}^{3} /\left(4 \pi G^{2}\left\langle M_{\mathrm{cl}}\right)^{2} \lambda n_{\mathrm{cl}}\right) \text {. }
$$

Here $R_{12}, P, M_{\mathrm{d}}, M,\left\langle M_{\mathrm{cl}}\right\rangle, n_{\mathrm{cl}}, \lambda \approx 10$ are the distance between stars, orbital period, dwarf and total mass in the binary, the average mass, the number density, and the Coulomb logarithm of the stars in the cluster.

When the dwarf fills its Roche lobe, the tightening of the binary leads to additional mass transfer due to conservation of the orbital angular momentum and the equation for the dwarf radius $R_{d}$

$$
R_{\mathrm{d}}=7.6 \times 10^{8}\left(\frac{2}{\mu_{\mathrm{e}}}\right)^{5 / 3}\left(\frac{M_{\odot}}{M}\right)^{1 / 3},
$$

for a nonrelativistic degenerate electron gas with a number of nucleons per electron equal to $\mu_{e}$. The loss of angular momentum in the collisions is equivalent to its loss by gravitational radiation, but in dense clusters it goes more rapidly and may lead to the loss of the companion during $t<\tau_{\mathrm{c}}$.

Let us make a quantitative estimation. If the dwarf fills its Roche lobe then $R_{12}$ is uniquely connected with $R_{d}$ and from eqs.(1-3) we get

$$
\tau_{\mathrm{s}}=10^{22} \frac{m m_{\mathrm{d}}}{m_{\mathrm{cl}}^{2} n_{51}}\left(\frac{\mu_{\mathrm{e}}}{2}\right)^{5 / 2} \mathrm{~s}
$$

Here $m=M / M_{\odot}, m_{\mathrm{d}}=M_{\mathrm{d}} / M_{\odot}, m_{\mathrm{cl}}=$ $\left\langle M_{\mathrm{cl}}\right\rangle / M_{\odot}, n_{51}=n_{\mathrm{cl}} / 10^{-51} \mathrm{pc}^{-3}$. The characteristic time of the loss of angular momentum by gravitational radiation in the same conditions may be written in the form

$$
\tau_{\mathrm{gr}} \approx \frac{10^{10}}{m^{2 / 3} m_{\mathrm{d}}^{11 / 3}}\left(\frac{2}{\mu_{\mathrm{e}}}\right)^{20 / 3} \mathrm{~s} .
$$

The ratio

$$
\frac{\tau_{\mathrm{gr}}}{\tau_{\mathrm{s}}} \approx 10^{-12} \frac{m_{\mathrm{cl}}^{2} n_{51}}{m^{5 / 3} m_{\mathrm{d}}^{14 / 3}}\left(\frac{2}{\mu_{\mathrm{e}}}\right)^{55 / 6},
$$

is equal to unity when the mass of the dwarf is equal to $m_{\mathrm{d}}=m_{\mathrm{d} 1}$ where

$$
m_{\mathrm{gr}} \approx 3 \times 10^{-3} \frac{m_{\mathrm{cl}}^{3 / 7} n_{51}^{3 / 14}}{m^{5 / 14}}\left(\frac{2}{\mu_{\mathrm{e}}}\right)^{55 / 28} .
$$

Analytical estimates show that after cosmological time $\tau_{c}$ in the presence of gravitational radiation and mass exchange the dwarf mass will be $m_{\mathrm{dc}}$

$$
m_{\mathrm{dc}}=4.5 \times 10^{-3} m^{-2 / 11}\left(2 / \mu_{\mathrm{e}}\right)^{20 / 11} \text {. }
$$


So if $m_{\mathrm{gr}}>m_{\mathrm{dc}}$ the collisions become more important than gravitational radiation at the time $t<\tau_{\mathrm{c}}$. This happens when the cluster parameters satisfy the relation

$$
n_{\mathrm{cl}}>3 \times 10^{5}\left(\frac{\mu_{\mathrm{e}}}{2}\right)^{15 / 22} \frac{m^{9 / 11}}{m_{\mathrm{cl}}^{2}} \mathrm{pc}^{-3} .
$$

This density is comparable with the density of compact globular clusters. The most characteristic cluster is M15 with a mass density $\rho=1.8 \times$ $10^{5} \mathrm{M}_{\odot} \mathrm{pc}^{-3}$ where 5 recycled pulsars (see table 1 ) have been discovered and 4 of them are single. The binary pulsar in this cluster has a neutron star as a companion, so its disruption according to eq.(4) is very difficult. When the period of the binary exceeds $\sim 8$ hours, interactions with the stars in the cluster may be more important for losing of angular momentum than gravitational radiation. Using the Keplerian velocity of the dwarf

$$
v_{\mathrm{d}}=\frac{2 \pi R_{12}}{P}=\left(\frac{2 \pi G M}{P}\right)^{1 / 3}
$$

in eq.(2) we obtain

$$
\tau_{\mathrm{s}}=\frac{M}{2 G\left\langle M_{\mathrm{cl}}\right)^{2} \lambda n_{\mathrm{cl}} P}=\frac{3.5 \times 10^{18} \mathrm{~m}}{m_{\mathrm{cl}}^{2} n_{5} P_{8}} \mathrm{~s},
$$

with $n_{5}=n_{\mathrm{cl}} / 10^{5} \mathrm{pc}^{-3}, P_{8}=P / 8$ hours. It is clear from eq.(11) that in very dense clusters $n_{5}>2$, this process may be important for decreasing the period of the binary up to about one day and even less during the time $\tau_{\mathrm{c}}$.

\section{Observational consequences}

\section{Nebulæ around evaporated pulsars}

The companions of eclipsing pulsars evaporate under the action of the pulsar beam radiation ejecting the matter which may form a surrounding envelope. Such an envelope has probably been observed around the first eclipsing pulsar (Kulkarni and Hester 1988). The same type of envelope may exist around the second eclipsing pulsar (Lyne et al. 1990b).

\section{The properties of LMXB in globular clusters}

The presence of several recycled pulsars in dense globular clusters shows that it is necessary to take into account the birth rate of LMXBs by tidal capture. The number of recycled pulsars in globular clusters must not be less then the total number of present and former LMXBs. Calculations give a number of tidally formed LMXBs not more than one per cluster (Bisnovatyi-Kogan and Romanova 1983). The existence of recycled pulsars implies restrictions on the lifetime of the active phase of LMXBs and their birth rate.

\section{On the decay of magnetic fields in neutron stars}

The theoretical estimations of the time of magnetic field decay depend on the depth of the currents and differ by many orders of magnitude. Statistical investigation of field decay has been done by Radhakrishnan (1982). One may do some rough estimations using observational data. The value of magnetic field measured by $\dot{P}$ is known for 293 pulsars from the list of Manchester and Taylor (1981). 179 pulsars from this list have $B>10^{12} \mathrm{G}$ and only 3 have $B<10^{11} \mathrm{G}$. For 46 pulsars with the age $\tau=P / 2 \dot{P}>10^{7.5}$

,y the number of pulsars with $B>10^{12} \mathrm{G}$ is only 2 and all 13 pulsars with $\tau>10^{8}$ y have $B<10^{12} \mathrm{G}$. These results show that during the pulsar lifetime the pulsar magnetic field decreases about an order of magnitude but not much more. This conclusion has been reached by Kulkarni (1986) [see also Srinivasan (1989)] after optical identification of pulsars PSR 0655+64 and PSR 0820+02 (see table 1).

The magnetic field of all recycled (without doubt) pulsars are 1-3 orders of magnitude smaller than the smallest magnetic field of ordinary radio pulsars. It is an indication of the action of an additional damping mechanism connected with accretion (Bisnovatyi-Kogan and Komberg 1974). The pressure of the falling plasma may rapidly ( $\sim 1$ day) screen the magnetic field of the neutron star in the absence of instabilities (Bisnovatyi-Kogan 1979).

The observations of absorption features in $\gamma$-ray bursts, Her X-1 and two other X-ray sources is often interpreted as a cyclotron absorption line, implying that the value of magnetic field in these objects is 3 to $6 \times 10^{12} \mathrm{G}$. If we take into account that $\gamma$-ray bursts belong to the oldest population of neutron stars with age $\tau>10^{\circ}$ years and Her X-1 is a strong accreting pulsar, then this interpretation contradicts the previous consideration. It is impossible now to make definite conclusions, but the most natural explanation would be different, not the cyclotronic nature of these absorption features. For the $\gamma$-ray bursts it could be absorption in the expanding cloud by heavy elements like I or $\mathrm{Ba}$, formed in the fission reaction which produces the $\gamma$-ray bursts. Polarization measurements of the feature in Her X-1 could help to solve this problem. 